\title{
Remark on the matrix element of $O_{11}$
}

\author{
Xiao-Gang He \\ Department of Physics, National Taiwan University, Taipei, Taiwan 10674 \\ and School of Physics, University of Melbourne, Parkville, Victoria, Australia 3052 \\ G. Valencia \\ Department of Physics and Astronomy, Iowa State University, Ames, Iowa 50011
}

(Received 16 September 1999; published 28 April 2000)

\begin{abstract}
The gluon dipole operator $O_{11}$ has received much attention recently because it can have a large coefficient in some SUSY extensions of the standard model. We estimate the matrix element of $O_{11}$ using naive dimensional analysis and find that, numerically, it is very close to the commonly used result of Bertolini, Eeg, and Fabbrichesi. We do not find any evidence to suggest that the latter is an underestimate.
\end{abstract}

PACS number(s): 12.39.Fe

One of the operators in the low energy effective Hamiltonian responsible for $|\Delta S|=1$ weak decays is the gluon dipole operator, which, within the standard model, can be written as $\left(H_{e f f} \equiv C_{11} O_{11}\right)$

$$
H_{e f f}=C_{11} \frac{g_{s}}{8 \pi^{2}} \bar{s}\left[m_{d} R+m_{s} L\right] T^{a} G_{a}^{\mu \nu} \sigma_{\mu \nu} d+\text { H.c., }
$$

where $G_{a}^{\mu \nu}$ is the gluon field strength tensor, and $L, R \equiv(1$ $\left.\mp \gamma_{5}\right) / 2$. In the standard model, the coefficient $C_{11}$ is sufficiently small to make the effects of this operator negligible. Beyond the standard model, however, this operator can have a large coefficient [1] and it becomes important to estimate its matrix element. The operator has received much attention recently, in connection with $\epsilon^{\prime} / \epsilon[2,3]$. To estimate the effect that this operator would have on $\epsilon^{\prime} / \epsilon$ within certain supersymmetric theories Ref. [3] allows the matrix element to be between one and five times as large as that calculated by Bertolini, Eeg and Fabbrichesi [4]. This matrix element appears in other calculations, such as that of $C P$ violation in hyperon decay [5], and for this reason it is appropriate to investigate its uncertainty.

The importance of this operator for kaon decays, and in particular for the analysis of $\epsilon^{\prime} / \epsilon$ dates back to the Weinberg model of $C P$ violation in the early 1980s. It was shown back then, that one had to pay particular attention to the chiral properties of the operator [6] in order to obtain physical amplitudes that obeyed the Feinberg-Kabir-Weinberg (FKW) theorem [7].

The gluon-dipole operator transforms as $(\overline{3}, 3)$ or $(3, \overline{3})$ under chiral rotations and, in the standard model, it is proportional to the light quark masses. It is well known that there are no operators with these transformation properties in the lowest order, $\mathcal{O}\left(p^{2}\right)$, weak chiral Lagrangian [8,9]. At next to leading order, $\mathcal{O}\left(p^{4}\right)$, there are several operators with the desired properties.

The strong interactions of pions and kaons are described to order $\mathcal{O}\left(p^{4}\right)$ in chiral perturbation theory by the Lagrangian of Gasser and Leutwyler [10]. The ingredients to construct this Lagrangian are the non-linear function $\Sigma$ $=\exp (2 i \phi / f)$ which contains the octet of pseudo Goldstone bosons $\phi$ and that transforms as $\Sigma \rightarrow R \Sigma L^{\dagger}$ under $S U_{L}(3)$ $\times S U_{R}(3)$. Interactions that respect chiral symmetry are con- structed in terms of derivatives of $\Sigma$. Explicit chiral symmetry breaking due to the non-zero light quark mass matrix $M=\operatorname{diag}\left(m_{u}, m_{d}, m_{s}\right)$ is introduced through the factors $S$ and $P$,

$$
\begin{aligned}
& S=\chi^{\dagger} \Sigma+\Sigma^{\dagger} \chi, \\
& P=i\left(\chi^{\dagger} \Sigma-\Sigma^{\dagger} \chi\right) .
\end{aligned}
$$

For our present purpose it suffices to take $\chi=2 B_{0} M$, where the parameter $B_{0}$ is proportional to the quark condensate $\langle\bar{q} q\rangle$, and relates the current quark masses to the meson masses:

$$
\langle\bar{q} q\rangle=-f_{\pi}^{2} B_{0}, \quad m_{K}^{2}=B_{0}\left(m_{s}+m_{d}\right), \quad m_{\pi}^{2}=B_{0}\left(m_{u}+m_{d}\right) .
$$

The leading order weak chiral Lagrangian transforming as $\left(8_{L}, 1_{R}\right)$ under chiral symmetry was first written down by Cronin [11] and has only one term (we use the notation $L_{\mu}$ $\left.=i \Sigma^{\dagger} D_{\mu} \Sigma\right)$

$$
\mathcal{L}_{W}^{(2)}=c_{2} \operatorname{Tr}\left(\lambda_{6} L^{2}\right)
$$

To introduce explicit chiral symmetry breaking due to the non-zero light quark mass matrix $M$ into the chiral Lagrangian one pretends that the mass matrix transforms as $M$ $\rightarrow R M L^{\dagger}$ and constructs operators with the desired transformation properties. For the dominant $\left(8_{L}, 1_{R}\right)$ weak operators it is well known that there is no mass term at order $p^{2}$, in accordance with the FKW theorem.

The operators that occur at next to leading order in the weak chiral Lagrangian have been written down in Ref. [9]. From their general list, those that could correspond to chiral realizations of the operator $O_{11}$ are the ones that contain one factor of the quark masses, they are (only five are independent) [9]

$$
\begin{aligned}
\mathcal{L}_{W}^{(4)}= & E_{10} \operatorname{Tr}\left(\lambda_{6}\left\{S, L^{2}\right\}\right)+E_{11} \operatorname{Tr}\left(\lambda_{6} L_{\mu} S L^{\mu}\right) \\
& +E_{12} \operatorname{Tr}\left(\lambda_{6} L_{\mu}\right) \operatorname{Tr}\left(\left\{L^{\mu}, S\right\}\right) \\
& +E_{13} \operatorname{Tr}\left(\lambda_{6} S\right) \operatorname{Tr}\left(L^{2}\right)+E_{14} \operatorname{Tr}\left(\lambda_{6} L^{2}\right) \operatorname{Tr}(S) \\
& +E_{15} \operatorname{Tr}\left(\lambda_{6} i\left[P, L^{2}\right]\right) .
\end{aligned}
$$


From the point of view of the transformation properties of $O_{11}$ under chiral symmetry all these terms are equally valid.

Reference [4] proceeds by noticing that the quark masses $m_{d}$ and $m_{s}$ in the short distance operator in Eq. (1) appear in a very specific form, depending on whether one considers a $|\Delta S|=1$ transition with a left-handed or a right-handed $s$-quark. They note that this requirement is satisfied by demanding that $\lambda_{6}$ and the quark mass matrix appear next to each other. For this reason, within the standard model, the list of possible operators in Eq. (5) reduces to the ones multiplying $E_{13}$ and the combination $E_{10}-E_{15}$. They further drop the operator multiplying $E_{13}$ because, in their model, it is suppressed by powers of $\alpha_{s} / \pi$.

We will, instead, treat all the operators that appear in Eq. (5) as valid chiral representations of $O_{11}$ for the following reasons. First, the operator is of interest only when it is generated with a large coefficient, and this happens beyond the standard model. In this more general case, when the operator is proportional to light quark masses, they do not always appear as in Eq. (1). For example, left-right models may have any quark mass associated with both left and righthanded $s$ quark fields [12]. Second, in the matrix element of $O_{11}$, the $E_{13}$ term is enhanced with respect to $E_{10}-E_{15}$ by $m_{K}^{2} / m_{\pi}^{2}$. Whereas this enhancement is always there, the suppression of $E_{13}$ argued in Ref. [4] is model dependent. Finally, there are cases where the operator is not proportional to light-quark masses, we discuss them in the last section.

Using Eq. (5) we find, for the matrix element of $O_{11}$,

$$
\begin{aligned}
C_{11}\left\langle\pi^{+} \pi^{-}\left|O_{11}\right| K^{0}\right\rangle= & -\frac{2 \sqrt{2}}{f_{\pi}^{3}}\left(m_{K}^{2}-m_{\pi}^{2}\right)\left(m_{\pi}^{2}\left(E_{10}-E_{15}\right)\right. \\
& +\left(m_{K}^{2}+m_{\pi}^{2}\right)\left(E_{10}+E_{15}\right)+m_{\pi}^{2} E_{11} \\
& +\left(4 m_{\pi}^{2}-2 m_{K}^{2}\right) E_{13} \\
& \left.+\left(m_{\pi}^{2}+2 m_{K}^{2}\right) E_{14}\right) .
\end{aligned}
$$

From this expression it is clear that the matrix element, in general, does not vanish in the limit $m_{\pi} \rightarrow 0$.

Although the framework of Eq. (5) is completely general, it does not tell us the size of the coefficients $E_{i}$. Since we do not have a detailed dynamical model to calculate them we use naive dimensional analysis as an estimate [13]. We are interested in terms that are proportional to $m_{K}^{2}$ and dimensional analysis is the same for all of them. For example, the operator proportional to $E_{10}$ has a term (other terms are related to this one by soft pion theorems)

$$
\mathcal{L}=g_{M} m_{K}^{2} \partial_{\mu} K \partial^{\mu} \pi+\cdots
$$

where, expanding Eq. (5), $g_{M}=4 \sqrt{2} E_{10} / f_{\pi}^{2}$. We find the reduced coupling using Weinberg's prescription [13],

$$
\left(g_{M}\right)^{r}=g_{M}(4 \pi)^{(2-N)}\left(4 \pi f_{\pi}\right)^{(D-4)}
$$

with $N=2$ fields and dimension $D=6$ and set it equal to the reduced coupling of the short distance operator $c m_{s} \bar{s} \sigma_{\mu \nu} G^{a \mu \nu} T^{a} d$ to find

$$
\left(E_{10}\right)_{N D A} \sim \frac{f_{\pi}^{2}}{4 \sqrt{2}} \frac{C_{11}}{8 \pi^{2}} \frac{g_{s}}{4 \pi} .
$$

From this we obtain,

$$
\left(C_{11}\left\langle\pi^{+} \pi^{-}\left|O_{11}\right| K^{0}\right\rangle\right)_{N D A} \sim\left(m_{K}^{2}-m_{\pi}^{2}\right) \frac{m_{K}^{2}}{f_{\pi}} \frac{C_{11}}{16 \pi^{2}} \frac{g_{s}}{4 \pi} .
$$

To evaluate this expression one would use a value of $g_{s}$ $\sim \sqrt{4 \pi}$. Note that an equivalent expression is obtained if one uses the term proportional to $E_{13}$ in Eq. (6) instead (or any other proportional to $m_{K}^{2}$ ).

\section{BAG MODEL ESTIMATE}

To check that our estimate based on naive dimensional analysis has the correct order of magnitude, we can directly compare it with the matrix element of $O_{11}$ in the MIT bag model. An explicit calculation of $\left\langle\pi\left|O_{11}\right| K\right\rangle$ within the MIT bag model exists in the literature [14]. If this is supplemented with a soft pion theorem, we can relate it to the matrix element we want, as was first done by Donoghue and Holstein [6],

$\left(C_{11}\left\langle\pi^{+} \pi^{-}\left|O_{11}\right| K^{0}\right\rangle\right)_{M I T}=-C_{11} \frac{g_{s} m_{s}}{32 \pi^{2}} \frac{A_{K \pi}}{2 f_{\pi}} \frac{m_{K}^{2}}{\Lambda^{2}}$.

Equation (11), is a trivial rescaling of the actual calculation in Ref. [6]. The factor $A_{K \pi}$ is obtained numerically from the Bag model, $A_{K \pi}=0.4 \mathrm{GeV}^{3}$, and the last factor in Eq. (11) introduces the suppression required by the FKW theorem. In accordance with power counting they choose $\Lambda \sim 1 \mathrm{GeV}$, corresponding to a matching of $O_{11}$ into an $\mathcal{O}\left(p^{4}\right)$ chiral Lagrangian of the form of Eq. (5) with an amplitude proportional to $m_{K}^{2}$. Numerically, we find that

$$
\left(C_{11}\left\langle\pi^{+} \pi^{-}\left|O_{11}\right| K^{0}\right\rangle\right)_{M I T} \approx 0.8\left(C_{11}\left\langle\pi^{+} \pi^{-}\left|O_{11}\right| K^{0}\right\rangle\right)_{N D A}
$$

in agreement with naive dimensional analysis.

\section{CHIRAL QUARK MODEL ESTIMATE}

More recently, Bertolini, Eeg and Fabbrichesi have used a chiral quark model supplemented with some matching conditions to estimate that [4]

$$
\begin{aligned}
\left(C_{11}\left\langle\pi^{+} \pi^{-}\left|O_{11}\right| K^{0}\right\rangle\right)_{C Q M}= & \frac{\sqrt{2}}{f_{\pi}^{3}}\left(m_{s}-m_{d}\right) m_{\pi}^{2} \\
& \times \frac{C_{11}}{16 \pi^{2}}\left(-\frac{11}{4}\langle\bar{q} q\rangle_{G}\right) .
\end{aligned}
$$

This is the expression that has been used in the recent literature $[2,3]$ and that we wish to contrast with our dimensional analysis estimate. The last factor in Eq. (13) is the model dependent quantity that arises from the chiral quark model, and that we take at face value. The factor of $m_{\pi}^{2}$ arises from 
the requirement that the short distance operator $O_{11}$ match into an order $p^{4}$ weak chiral Lagrangian of the form proportional to $E_{10}-E_{15}$ [4]. As mentioned above, the requirement that the quark mass matrix $M$ and $\lambda_{6}$ appear next to each other selects this term plus the one whose coefficient is $E_{13}$. The latter is dropped in Ref. [4] because all products of two traces are suppressed in their model.

The specific numerical result of Ref. [4], however, is very similar to our dimensional analysis estimate. This happens because the $m_{\pi}^{2} / m_{K}^{2}$ suppression that occurs in the amplitude from the operators selected by their matching is compensated by the large numerical coefficient, 11, in Eq. (13). Numerically,

$$
\left(C_{11}\left\langle\pi^{+} \pi^{-}\left|O_{11}\right| K^{0}\right\rangle\right)_{C Q M} \approx 1.4\left(C_{11}\left\langle\pi^{+} \pi^{-}\left|O_{11}\right| K^{0}\right\rangle\right)_{N D A} .
$$

We see that all three estimates are numerically very similar and in agreement with each other within the uncertainty of each approach. However, it is clear that the numerical agreement with the chiral quark model result of Ref. [4] is accidental.

As we have mentioned, the operator $O_{11}$ is of great interest in certain theories beyond the standard model. For example, in supersymmetry it could give a large contribution to $\epsilon^{\prime}$. In that context, Ref. [3] has used the matrix element of Ref. [4]. Noting the $m_{\pi}^{2}$ suppression, however, they have allowed for the matrix element to be between one and five times as large as the chiral quark model estimate [4]. From our dimensional analysis, we conclude that the matrix element is not likely to be much larger than that of Ref. [4], as seen in Eq. (14), and for this reason we prefer to assign a factor of two uncertainty in either direction to the result Eq. (10) $[5]$.

\section{$O_{11}$ WITHOUT LIGHT QUARK MASSES}

In all the cases that we have discussed, we have considered the matrix element of the operator written as in Eq. (1). In this form it appears that the operator vanishes in the chiral limit being proportional to the light-quark masses. This was, in fact, an important ingredient in the matching to a corresponding chiral Lagrangian. However, in some models of interest, this is just an artifact of the normalization. In such cases it is better to write

$$
H_{e f f}=\widetilde{C}_{11} \widetilde{O}_{11}+\text { H.c., }
$$

where $\quad \widetilde{O}_{11}=\left(g_{s} / 8 \pi^{2}\right) \bar{s}[R, L] T^{a} G_{a}^{\mu \nu} \sigma_{\mu \nu} d$. Here $\quad[R, L]$ means that both chiralities are possible. In this case we want to construct a low energy meson Lagrangian that transforms as $(\overline{3}, 3)$ or $(3, \overline{3})$ but that is not proportional to the light quark masses.

It is possible to write a term without derivatives,

$$
\mathcal{L}=\operatorname{Tr}\left(\Sigma^{\dagger} h+h^{\dagger} \Sigma\right)
$$

that does not contribute to $K \rightarrow \pi \pi$ amplitudes once tadpoles are properly subtracted. Here the matrix $h$ is equal to $h$ $=\left(\lambda_{6}+i \lambda_{7}\right) / 2$ whose only non-zero entry is $h_{23}=1$. The leading order amplitude arises from a Lagrangian with two derivatives. In terms of the matrix $h$ we can write, for example,

$$
\begin{aligned}
\mathcal{L}= & g_{N 1} \operatorname{Tr}\left(h D_{\mu} \Sigma^{\dagger} D^{\mu} \Sigma \Sigma^{\dagger}\right) \\
& +g_{N 2} \operatorname{Tr}\left(\Sigma^{\dagger} h+h^{\dagger} \Sigma\right) \operatorname{Tr}\left(D_{\mu} \Sigma^{\dagger} D^{\mu} \Sigma\right)
\end{aligned}
$$

The first term in Eq. (17) yields a matrix element proportional to $m_{\pi}^{2}$, but the second term does not. We find

$$
\widetilde{C}_{11}\left\langle\pi^{+} \pi^{-}\left|\widetilde{O}_{11}\right| K^{0}\right\rangle=g_{N 2} \frac{2 \sqrt{2}}{f_{\pi}^{3}} m_{K}^{2}+\mathcal{O}\left(m_{\pi}^{2} / m_{K}^{2}\right) .
$$

Although we cannot compute $g_{N 2}$, we can again estimate it with naive dimensional analysis following Weinberg. Noting that the operator does not contain terms with only two fields, we find

$$
g_{N 2} \sim f_{\pi}^{3}\left(\frac{g_{s} \widetilde{C}_{11}}{16 \pi^{2}}\right)
$$

resulting in

$$
\left(\widetilde{C}_{11}\left\langle\pi^{+} \pi^{-}\left|\widetilde{O}_{11}\right| K^{0}\right\rangle\right) \sim m_{K}^{2} \frac{g_{s} \widetilde{C}_{11}}{8 \pi^{2}}
$$

This result is equivalent to Eq. (10), differing only by factors of order one that cannot be accounted for with naive dimensional analysis.

In conclusion, we find that the best guess that exists at present for the matrix element of $O_{11}$ is Eq. (10). This estimate is based on naive dimensional analysis and is in agreement with an MIT bag model estimate. It is also in accidental numerical agreement with the chiral quark model estimate of Bertolini, Eeg and Fabbrichesi that is specific to the standard model case. Until there exists a reliable way to compute the matrix element, results such as Eq. (10) should be viewed with caution. We do not find any evidence to support the bias of Ref. [3] of assigning an uncertainty to this matrix element only in the direction in which it gets larger.

The work of G.V. was supported in part by DOE under contract number DEFG0292ER40730. The work of X-G.H. was supported by NSC of R.O.C. under grant number NSC88-2112-M-002-041 and Australian Research Council. G.V. thanks the theory group at SLAC for their hospitality and for partial support while this work was completed. We thank S. Bertolini, J. Eeg, M. Fabbrichesi and H. Murayama for useful comments. 
[1] A. Kagan, Phys. Rev. D 51, 6196 (1995).

[2] D. Chang, X.-G. He, and B. McKellar, hep-ph/9909357; G. Eyal et al., J. High Energy Phys. 11, 032 (1999); B. Holdom, Phys. Rev. D 61, 011702 (2000); S. Baek et al., hep-ph/9907572; A. Masiero and H. Murayama, Phys. Rev. Lett. 83, 907 (1999).

[3] A. Buras et al., hep-ph/9908371.

[4] S. Bertolini, J. Eeg, and M. Fabbrichesi, Nucl. Phys. B449, 197 (1995).

[5] X.-G. He et al., Phys. Rev. D (to be published), hep-ph/9909562.

[6] J. Donoghue and B. Holstein, Phys. Rev. D 32, 1152 (1985).
[7] G. Feinberg, P. Kabir, and S. Weinberg, Phys. Rev. Lett. 3, 527 (1959).

[8] J. Bijnens and M. Wise, Phys. Lett. 137B, 245 (1984).

[9] J. Kambor, J. Missimer, and D. Wyler, Nucl. Phys. B346, 17 (1990).

[10] J. Gasser and H. Leutwyler, Nucl. Phys. B250, 465 (1985).

[11] J. A. Cronin, Phys. Rev. 161, 1483 (1967).

[12] X.-G. He and G. Valencia, Phys. Rev. D (to be published), hep-ph/9908298.

[13] H. Georgi and L. Randall, Nucl. Phys. B276, 241 (1986); S. Weinberg, Phys. Rev. Lett. 63, 2333 (1989).

[14] J. Donoghue, J. Hagelin, and B. Holstein, Phys. Rev. D 25, 195 (1982). 